\title{
Role of Hepatitis B Virus Large Surface Protein in Promoting Activation of Nuclear SREBPs via ER Stress: Implications for Hepatocellular Carcinoma
}

\author{
Subir Ghosh ${ }^{1}$ \\ ${ }^{1}$ Division of Natural Sciences, College of Natural and Applied Sciences, University of Guam, United States \\ Correspondence: Subir Ghosh, Division of Natural Sciences, College of Natural and Applied Sciences, \\ University of Guam, United States. E-mail: sghosh@triton.uog.edu
}

Received: October 24, 2018

Accepted: November 20, 2018

Online Published: February 10, 2019

doi:10.5539/ijb.v11n2p13

URL: https://doi.org/10.5539/ijb.v11n2p13

\begin{abstract}
One of the most common fatal malignancies in the world is Hepatocellular carcinoma (HCC) and hepatitis B virus (HBV) infection has been identified as a leading risk factor for HCC. Studies have suggested that during infection the large surface viral protein of HBV (LS protein) is targeted to the endoplasmic reticulum (ER) of the host liver cell, where its accumulation causes ER stress leading to HCC. The LS protein is also believed to stimulate the lipogenic activity of the ATP citrate lyase (ACL) enzyme, leading to excessive synthesis of lipids required for the rapidly dividing liver tumor cell. Here, I propose a hypothesis linking viral LS protein-induced ER stress to generation of the mature transcriptionally active nuclear form of sterol regulatory element-binding protein (nSREBP). nSREBP translocates to the nucleus and binds to sterol regulatory elements (SRE) present in the ATP citrate lyase (ACL) gene promoter, thereby elevating ACL transcription levels and overproduction of the ACL enzyme protein. Inhibition of LS protein activity in the ER may provide a therapeutic strategy in treating HBV induced HCC.
\end{abstract}

Keywords: Hepatitis B Virus Large Surface Protein, Hepatocellular Carcinoma, ER Stress, ATP Citrate Lyase, SREBP

\section{Introduction}

Hepatitis B virus (HBV) infection has been identified as a major cause for Hepatocellular carcinoma (HCC) in chronically infected patients (Neuveut et al., 2010; Xu et al., 2014; Ringehan et al., 2017; Mani \& Andrisani, 2018). Estimates indicate that $53 \%$ of HCC cases worldwide are HBV related. The mechanism by which HBV induces tumor formation in liver is still not well understood and provides an important model for understanding the molecular pathogenesis of HCC and for the development of novel anti-viral drugs (Churin et al., 2015; Levrero \& Zuckman-Rossi, 2016; Lamontagne et al., 2016). It has been shown that the risk for HBV infected patients developing HCC is directly correlated with increased levels of viral DNA replication as assayed by HBV-DNA serum levels (Chen et al., 2006; Chen \& Yang, 2011; Bauer et al., 2011). The HBV DNA genome encodes the structural core and surface envelope proteins, the viral polymerase/reverse transcriptase, and the regulatory $\mathrm{X}$ protein $(\mathrm{HBx})$. $\mathrm{HBx}$ has been the major focus of investigations since it has been shown to be a oncoprotein (Neuveut et al., 2010; Bouchard \& Navas-Martin, 2011; Riviere et al., 2014; Lamontagne et al., 2016). The viral genome encoded surface proteins have also been implicated in the progression of HCC and the mechanism by which they induce HCC is a subject of ongoing investigation. There are three HBV viral surface proteins: small, middle, and large (LS), encoded by a single open reading frame in the HBV viral genome - the three surface proteins are synthesized from three different translation initiation codons within the open reading frame (Lamontagne, 2016). The LS protein is thought to play key roles in binding to host receptors in the human liver and in the assembly of the virion and its release from the cell (Churin et al., 2015). The three surface proteins differ in their domain organization (Pollicino et al., 2014; Churin et al., 2015). The LS protein is composed of three domains: the $\mathrm{N}$ - terminal preS1 domain, a central preS2 domain and a C-terminal S domain. The middle surface protein contains only the preS2 and the $\mathrm{S}$ domain while the small surface protein contains only the C-terminus S domain. The preS1 domain contains108, 118, or 119 amino acids, depending on the viral genotype. The preS2 domain is 55 amino acids long and the $\mathrm{S}$ domain contains 226 amino acids. The Hepatitis B viral large surface protein (LS) has been shown to have oncogenic potential when expressed in transgenic mice, 
in the absence of the entire HBV genome (Chisari et al., 1989). Thus the LS protein may also play a significant role in the induction of HCC. In an infected liver cell, the Endoplasmic Reticulum (ER) is where the viral surface proteins are synthesized and become inserted into the ER membrane becoming transmembrane proteins. Accumulation of surface proteins in the ER leads to ER stress and hepatic cell injury initiating a sequence of events leading to HCC (Churin et al., 2015; Lamotagne et al., 2016). It was also found that the serum and livers of patients with chronic HBV infection had significant levels of a mutant large surface protein with an in-frame deletion in the preS2 region, S2-LHBs (Su et al., 2014). Different investigative groups have investigated effects of preS domain mutants and truncated forms of the surface proteins in liver cell culture and transgenic mice studies and report that these mutant surface proteins induce ER stress and activate a variety of signaling pathways, for example, activation of protein kinase C-dependent c-raf-1/MAP1-kinase signaling pathway, mammalian target of rapamycin (mTOR) signal pathway, and even non-ER stress induced signaling pathways leading to host genomic instability and HCC (Pollicino et al., 2014; Li et al., 2016; Teng et al., 2017). Interestingly, in a study of HCC patients, analysis of liver biopsies of tumor tissue showed higher expression of the nuclear sterol regulatory-binding protein 1 (SREBP-1) transcription factor compared to that of adjacent non-tumor liver tissue ( $\mathrm{Li}$ et al., 2014). SREBPs are well studied transcription factors involved in lipid biosynthesis (Horton et al., 2002) and target a variety of lipogenic genes including the ATP citrate lyase gene which has been shown to be critical for liver tumor formation (Cheng et al., 2018). The precise mechanism by which the LS protein via ER stress results in the enhanced generation of the mature transcriptionally active nuclear form of SREBP1 transcription factor is not known. Researchers are in agreement that liver tumors caused by HBV are extremely heterogeneous exhibiting a complex and wide genetic/epigenetic landscape and involving multiple transcription and signaling pathways (Levrero and Zucman-Rossi, 2016).

Hepatitis B virus may use multiple strategies to lead to HCC and the LS protein may be an important player in promoting malignant transformation of the liver and deserves further investigation. Here, I focus on the viral LS protein and suggest a plausible mechanism by which it may promote HCC mediated by ER stress leading to the generation of the mature transcriptionally active nuclear form of the sterol receptor-binding protein (nSREBP) from the ER resident precursor inactive form of SREBP. The mature form, nSREBP translocates to the nucleus and upregulates transcription from target lipogenic genes including the ATP citrate lyase gene. The LS protein is a potential target for treating HBV-induced cancer and this hypothesis may lead to various possibilities for the development of potent inhibitors of LS protein activity.

\section{Oncogenic Potential of Hepatitis B Virus LS Protein}

Establishment of transgenic mice model systems have led to the suggestion that the LS envelope protein of HBV may be critical in promoting HCC in the human liver. Chisari et al., 1989, were the first to demonstrate that over-expression of the LS protein in liver of transgenic mice leads to chronic hepatitis and malignant transformation. It was found that in these transgenic mice over-expressing only the LS protein of HBV, there was accumulation of the LS protein in the ER of liver cells followed by chronic liver injury and an associated inflammatory response, chromosomal aneuploidy and transcriptional deregulation, ultimately leading to development of liver tumors. Liver injury induced by the LS protein results in an altered liver cell physiology creating a damaging cellular environment conducive to generation of random mutations and chromosomal alterations and aneuploidy, leading to deregulation of cell cycle control genes favoring liver cell transformation and proliferation. This transgenic mouse model system, where the mice are immunologically tolerant to the HBV surface protein, also ruled out the general belief that hepatic injury during HBV infection is due solely to cytotoxic T cell response to the HBV surface antigens displayed by the major histocompatibility complex at the surface of the infected liver cell. The transgenic mice experiment also revealed the complex and heterogeneous nature of HBV infection - the incidence and aggressiveness of the liver tumors caused by the overexpressed LS protein depended on the sex, age, and genetic background of the transgenic mice. Clearly the mechanism by which HBV infection leads to HCC may vary among individual patients infected by HBV. The oncogenic potential of the LS envelope protein was also confirmed by subsequent studies using transgenic mice (Churin et al., 2014). Recently, Song et al. (2018) created CRISPR/Cas9 knockouts of HBV preS1, preS2, and S open reading frame sequences stably integrated in the host cell genome in HBV infected HCC cells, HepG2-2.15. In these knockout cells, the level of viral surface proteins decreased and HCC proliferation in vitro was significantly reduced demonstrating the oncogenic function of the LS surface protein. They also showed that the surface protein knockout HCC cell lines when injected subcutaneously into nude mice exhibited significantly decreased potential for developing tumors in vivo. Song et al., 2018 further confirmed the oncogenic potential of the LS surface protein by demonstrating in vitro cell proliferation in HBV-negative HCC cell lines, SK-hep1 and HLF, that were stably transfected with a LS-FLAG tagged vector construct and expressing the LS protein. These 
LS expressing cell lines also developed in vivo into tumors when injected into nude mice.

\section{ER Stress Induced by Hepatitis B Virus LS Protein}

During virus infection the LS protein is cotranslationally inserted into the membrane of the ER and exists in the liver cell as a ER transmembrane protein. Several studies have shown that the LS protein of HBV which is targeted to the endoplasmic reticulum of the host liver cell, accumulates in the ER causing ER stress. However, the mechanism and pathway by which ER stress leads to HCC is not well understood. The relationship between ER stress and HBV infection and tumorigenesis of the liver deserves deeper investigation (Lazar et al., 2014). Liver biopsies from patients who have chronic HBV infection revealed strong hypertrophy of the ER. The altered ER structure was shown to result from the accumulation of large amounts of LS-containing filamentous particles within the hyperplastic ER and was termed "ground glass hepatocyte" and became a diagnostic for HBV related HCC (Hollinger, 1996; Lazar et al., 2014). Over-expression of LS protein in human HuH7 hepatoma cells in culture, either as an expression plasmid containing coding sequences for LS protein alone or in context to the entire HBV genome in an expression plasmid, resulted in ER stress (Xu et al., 1997). It was also found that accumulation of mutants of the LS surface protein in the ER of transgenic mice resulted in induction of ER stress followed by oxidative DNA damage and mutagenesis potentially resulting in host genomic instability leading to the development of HCC (Hsieh et al., 2004; Wang et al., 2006). Churin et al. (2014) investigated transgenic mice expressing HBV surface proteins, and found that the hepatocytes of one of the two mouse strains on BALB/c genetic background used in the study exhibited persistent ER stress which led to the activation of one of the three branches of the unfolded protein response (UPR) in response to ER stress, namely induction of PERK (protein kinase-like endoplasmic reticulum kinase) that led to increased pro-apoptotic C/EBP homologous protein (CHOP) expression. CHOP expression resulted in reduced tumorigenesis in these mice. In contrast, the second C57BL/6 transgenic strain which developed liver tumors did not show increased CHOP expression leading the authors to conclude that hepatic HBV surface protein expression induction of tumor development is dependent on the host genetic background. The cell proliferation favoring transcription factors c-jun and STAT-3 were also found to be activated in these transgenic mice. Montalbano et al. (2016) have shown that ER stress is induced in human liver cell lines, Huh-7 and HepG2 by overexpressing plasmids containing coding sequences for the HBV large surface protein. They found upregulation of typical ER stress markers chaperone $\mathrm{BiP} / \mathrm{GRP} 78$ proteins (Binding immunoglobulin protein/Glucose-related protein 78 ); the transcription factor CHOP/GADD153 (C/EBP homologous protein/Growth arrest and DNA damage inducible gene 153); phosphorylation of PERK (PKR-like ER Kinase) and EIF2a (Eukaryotic initiation factor 2a); and splicing of XBP1 (X box binding protein 1). ER stress has also been implicated in other cancers and lipid-related diseases (Wang et al., 2005; Xu et al., 2014; Lee et al., 2015; Riha et al., 2017).

The unfolded protein response (UPR) allows a cell to survive when ER stress is encountered and is responsible for the correction of proper folding of proteins or degradation and disposal of incorrectly folded proteins. If unable to correct the folding of proteins, the cell is eliminated via the apoptotic pathway (Riha et al., 2017). The mechanism by which the LS protein of HBV induces ER stress leading to the promotion of tumors in stark contrast to apoptosis is an active area of investigation, since it is a therapeutic target for treating HCC (Kim et al., 2017). Typically, viruses use the unfolded protein response (UPR) induced by ER stress to their own benefit in order to complete the viral life-cycle. However, it may also be possible that ER stress induced by viral proteins leads to activation of alternate cellular pathways leading to tumorigenesis. The mechanism by which the LS protein of Hepatitis B Virus promotes tumorigenesis is still unclear. The possibility of the LS protein taking advantage of ER stress to activate downstream signaling and transcription pathways resulting in the transcriptional activation of target genes in the nucleus leading to HCC is intriguing. It is imperative to identify specific target genes and elucidate specific ER stress induced pathways in hepatic cells, that will lead to novel therapeutic strategies to combat HBV induced HCC.

\section{ATP Citrate Lyase Activity in HBV Infected Liver Cells}

The mechanism by which the LS protein of HBV causes liver cell tumors is still a subject of intense investigation. There is a possibility that the viral LS protein may play a role in upregulating the activity of the ATP citrate lyase (ACL) enzyme in the infected host liver cell. ACL enzyme activity has been a subject of interest for understanding tumors in general and the ACL gene may be a downstream target of the viral LS surface protein. It is well known that many tumors display an elevated rate of aerobic glycolysis (glucose utilization) leading to excessive synthesis of lipids required for the rapidly dividing cancer cell (Hatzivassiliou et al., 2005; DeBerardinis \& Chandel, 2016; Cheng et al., 2018). Clinically, this fact is used in 18-F-2-deoxyglucose PET imaging for the detection of primary and metastatic tumors (Gambhir, 2002). High levels of glycolysis is advantageous to the proliferating tumor cells since it provides for the excess end-product 
pyruvate, which in turn is redirected to the biosynthesis of lipids. This excess lipid is required for membrane synthesis and synthesis of cellular macromolecules that is necessary for the rapidly dividing cancer cell. Reprograming of lipid metabolism is a hallmark of malignancy and lipid biochemical pathways is a target for anti-cancer strategies (Guo et al., 2013; Gabitova et al., 2013; Cheng et al., 2018). Significantly, Craig Thompson's cancer research laboratory knocked down endogenous ACL in a human lung adenocarcinoma cancer cell line using RNA interference (siRNA) or a chemical inhibitor and showed that the cells exhibited reduced proliferation and viability (Hatzivassiliou et al., 2005). They also observed that the same treatments in mice reduced in vivo tumor growth. Thus, glucose metabolism is linked to lipid synthesis, an important feature of cancer metabolism, and the key enzyme that is involved in this biochemical pathway is ATP citrate lyase (Zaidi et al., 2012; Granchi, 2018).

Evidence for enhanced ACL enzyme activity in HCC comes from studies performed in context to (i) liver cell biopsies obtained from HCC patients and HBV-infected HCC patients; (ii) in HBV preS2 mutant transgenic mice; (iii) in a HuH-7 hepatocyte derived carcinoma cell line that was originally taken from a liver tumor in a male human patient; and (iv) in HepG2 cell line derived from the liver tissue of a male human patient with HCC. Interestingly, the mRNA expression of lipogenic enzymes in human HCC samples from ten patients were examined and found to be significantly elevated, including the ACL mRNA, compared with surrounding liver tissue that did not show cancer (Yahagi et al., 2005). Western blot analysis of thirty chronic HBV-infected HCC patients revealed that in twenty of these patients, the HCC tissues had increased levels of ACL enzyme protein compared with paired adjacent non-tumor liver tissue (Teng et al., 2015). There has been reports of activation of ACL activity in Hepatitis B virus transgenic mouse liver. In a recent study, Teng et al., 2015 reported that in livers of transgenic mice expressing the pre-S2 deletion mutant of the LS protein of HBV, the ATP citrate lyase gene (ACL) mRNA levels was upregulated in HCC tissues. Western blotting also revealed the presence of higher levels of the ACL enzyme protein compared with the non-transgenic livers. These preS2 mutant transgenic mice also exhibited increased accumulation of lipids in the HCC tissues. The studies further showed that cell cultures of the human liver tumor derived HuH-7 cells transfected with a preS2 mutant exhibited higher levels of the ACL enzyme protein as analyzed by Western blotting and also increased proliferation compared with cells transfected with a control plasmid. In an earlier study, a cDNA microarray chip analysis of HBV transgenic mice liver revealed that genes in the lipid biosynthetic pathway including the ACL enzyme gene were upregulated (Hajjou et al., 2005). However, in these transgenic mice HBV was replicating in the liver and did not exhibit liver cytopathology. No follow up study was reported to see if these transgenic mice developed liver tumors. In another study, silencing of ACL enzyme in liver HepG2 cells (liver cancer derived) in culture as well as cell lines derived from other cancer types by short hairpin RNA led to growth arrest and apoptosis (Zaidi et al., 2012). These data taken together indicate that upregulated ACL enzyme activity may be involved in HBV-induced HCC. Since it has been convincingly shown that the wild-type LS protein has oncogenic potential by itself (Chisari et al., 1989), the possibility exists that the LS protein induces HCC via a specific pathway/s leading to upregulated lipogenic ACL activity.

\section{ER Stress and Sterol Regulatory-Binding Protein Activated Transcriptional Regulation of the ATP Citrate Lyase Gene}

The two striking features revealed by investigations involving HBV induced HCC as discussed above are induction of ER stress by the LS surface protein of HBV and upregulation of ACL activity. There is a possibility that the two events are linked with LS protein induced ER stress resulting in transcriptional upregulation of the ACL gene. The Sterol regulatory element-binding proteins (SREBPs) may provide the mechanistic link between the LS protein of HBV and upregulation of lipogenic ACL activity. SREBPs are a family of basic-helix-loop-helix leucine zipper transcription factors that are located in the membrane of the endoplasmic reticulum (ER) and play a central role in the biosynthesis of lipids in the cell (Horton et al., 2002; Cheng et al., 2018; Moslehi \& Hamidi-zad, 2018). There are three isoforms of SREBP, namely SREBP-1a, SREBP-1c, and SREBP-2. Sterol regulatory element-binding proteins (SREBPs) bind to the sterol regulatory element (SRE) of target gene promoters to effect an increase in expression of genes involved in lipid biosynthesis and uptake. Several studies have revealed that SREBPs in various cancers are dramatically upregulated resulting in rapid tumor growth and thereby, provide potential pharmacological targets for cancer therapy (Shao \& Epenshade, 2012; Cheng et al., 2018). Recent studies have also shown increased levels of SREBP in Hepatitis B Virus related HCC. SREBP-1 was found to be upregulated in liver biopsies from HCC human patients infected with HBV and shown to promote tumor progression ( $\mathrm{Li}$ et al., 2014). HCC tissues and matched normal tumor-adjacent tissues were collected from 39 human patients infected with HBV. In the HCC tissues of 27 of the 39 patients, both SREBP-1 mRNA and protein levels were significantly elevated as analyzed by qRT-PCR, 
Western blot \& Immunohistochemistry, respectively. The high expression level of SREBP-1 was found to be associated with large tumor size. Western blot analysis was also performed by Teng et al. (2015) on HBV-related HCCs taken from 30 human patients. Both SREBP-1 and ACL protein levels were significantly higher in HCC tissues compared with matched adjacent non-cancerous tissue for 20 of the 30 patient samples. Teng et al., 2015 also reported that in livers of transgenic mice expressing the pre-S2 deletion mutant of the LS protein of HBV, Western blot analysis showed both ACL and SREBP-1 were upregulated.

The ATP citrate lyase gene has been shown to be transcriptionally regulated by sterol regulatory element binding proteins (SREBP), in both cell culture transient transfection, and transgenic mice experiments. Overexpression of recombinant SREBP-1a and SREBP-2 proteins in transgenic mice liver resulted in elevated levels of ACL mRNA (Shimomura et al., 1998). In SREBP-1a gene knockout mice experiments, the hepatic mRNA levels of ATP citrate lyase enzyme was found to be suppressed (Shimano et al., 1999). Ameniya-Kudo et al. (2002) have also shown that SREBP mediates transcriptional activation of several lipogenic enzyme coding genes including the ATP citrate lyase gene in human hepatoma $\mathrm{HepG} 2$ cells in culture, using reporter gene assays and expression plasmids containing cDNA encoding for human nuclear forms of SREBP-1a and SREEBP-2. The human ACL gene promoter sequence was found to have three potential sterol regulatory element (SRE) sites placed within $-131 \mathrm{bp}$ upstream of the transcription start site (Figure 1), and gel mobility shift assays showed that recombinant SREBP proteins bind to the SRE sites (Sato et al., 2000). Sato et al. (2000) further found that the SREBP-1a isoform more strongly activated transcription compared to the SREBP-2 protein in CHO-487 cell culture transfection assays. The rat and human ATP citrate lyase promoters are highly conserved. Gel mobility shift assays revealed the presence in rat ATP citrate lyase promoter of four SREBP-1a binding sites within a 55-base pair upstream promoter sequence from -114 to -60 . Moon et al. (2000) transiently transfected luciferase reporter constructs of the rat ACL promoter in the presence or absence of expression plasmid constructs of the mature form of human SREBP-1a or SREBP-2 in cultured Alexander human hepatoma cell line. Overexpression of both forms of SREBP resulted in significant stimulation of the rat ATP citrate lyase gene promoter, with SREBP-1a showing higher activation than SREBP-2, just as was observed for the human ATP citrate lyase promoter.

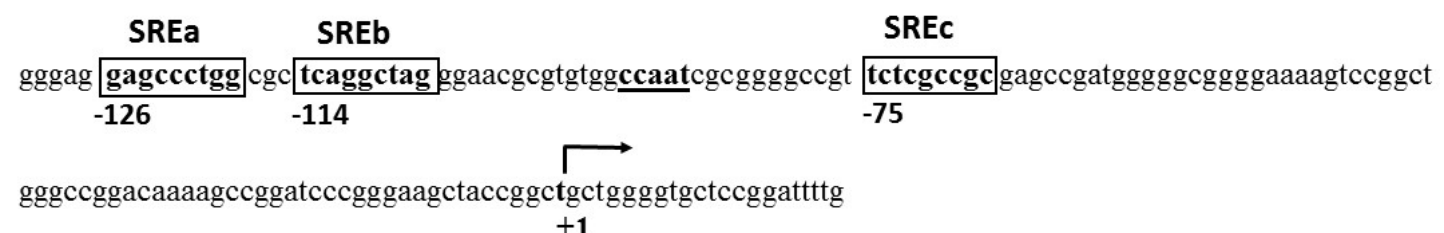

Figure 1. The human ATP citrate lyase promoter. The transcription start site is +1 . The three upstream sterol response element (SRE) sites are shown in bold and boxed. Nuclear sterol regulatory element-binding proteins (nSREBPs) activate transcription of ACL gene by binding to the SRE sites. The CAAT box (shown in bold and underlined) is recognized by the ubiquitous transcription factor, NF-Y

To summarize, recent studies indicate that ER stress is induced in chronic HBV infections and is believed to be due to the overexpression and accumulation of the HBV LS surface protein in the ER. A consequence of ER stress is the upregulation of SREBP transcription factor and ACL enzyme activity. However, there have been no reports of the precise mechanism by which the LS protein in the ER effects the release of the mature nuclear form of SREBP (nSREBP). It is important to elucidate the mechanism of LS action since inhibition of LS protein activity may provide a therapeutic strategy in treating HBV induced HCC. I have, therefore, as discussed below proposed a plausible hypothesis for the mechanism by which the LS protein of HBV interacts with the membrane bound inactive SREBP to produce the transcriptionally active nuclear form of SREBP.

\section{Hypothesis for the Role of LS Protein of HBV in Converting the ER Membrane-Bound Inactive SREBP Protein in the Liver to the Nuclear Transcriptionally Active Form of SREBP (nSREBP), Leading to Upregulation of the ATP Citrate Lyase Gene}

The investigative studies linking chronic HBV-infection and the LS viral envelope protein to the development of HCC have utilized a range of experimental systems including, liver cell biopsies obtained from HCC patients; transgenic mice liver expressing the LS surface envelope protein; hepatocyte cell cultures; as well as serological studies from human patients. The data taken together indicate that the LS protein of HBV induces ER stress in chronic HBV infected liver leading to upregulation of SREBP transcription factor and ATP citrate lyase (ACL) 
activity. However, the mechanism by which the accumulation of the LS protein in the ER of an HBV-infected liver cell exhibiting ER stress results in SREBP activation to form the nuclear transcriptionally active mature nSREBP is unknown.

The pioneering work in the laboratories of Noble Prize winners, Goldstein and Brown led to the elucidation of the regulation of lipid homeostasis in vertebrates by the SREBP family of transcription factors (Horton et al., 2002; Espenshade, 2006; Jacquemyn et al., 2017; Cheng et al., 2018). In the liver cells, the SREBPs are located as trans-membrane proteins in the ER. The SREBP transcription factors are present in the ER in an inactive form. Transcriptional activation of target genes in the nucleus by SREBP proteins first involves proteolytic processing of the ER membrane-bound inactive SREBPs to the transcriptionally active nuclear form, nSREBPs. The released nSREBPs enter the nucleus, mediated by a nuclear localization signal, to target at least thirty genes required for lipid and cholesterol biosynthesis, including the ACL gene. There are three proteins involved in processing of ER membrane bound immature SREBPs - one is an escort protein SCAP (SREBP-cleavage-activating protein) and the other two are Golgi resident proteases, S1P (site 1 protease) and $\mathrm{S} 2 \mathrm{P}$ (site 2 protease). In the ER membrane, the $\mathrm{COOH}$-terminal domain of SREBP is bound via protein-protein interactions to the COOH-terminal domain of SCAP. The NH2-terminal domain of SCAP in turn is bound to the NH2-terminal domain of the insulin-induced gene protein (Insig), which is also anchored to the ER. The Insig protein contains six transmembrane domains. The Insig/SCAP/SREBP complex prevents SREBP from exiting the ER maintaining the SREBP protein as the precursor inactive form. When cellular cholesterol levels fall, SCAP dissociates from Insig and serves as an escort moving the SREBP from the ER to the Golgi apparatus. In the Golgi are located the S1P and S2P proteases which sequentially processes the precursor inactive SREBP protein resulting in the release from the membrane of the NH2-terminal basic helix-loop-helix leucine zipper (bHLH-LZip) transcriptional activation domain of SREBP which constitutes the mature transcriptionally active form, nSREBP. The bHLH-LZip also contains a nuclear localization signal (Eberle et al., 2004). Translocation of nSREBP to the nucleus results in transcriptional activation of target genes by binding to sterol response elements (SREs) in the promoter/enhancer regions of target genes.

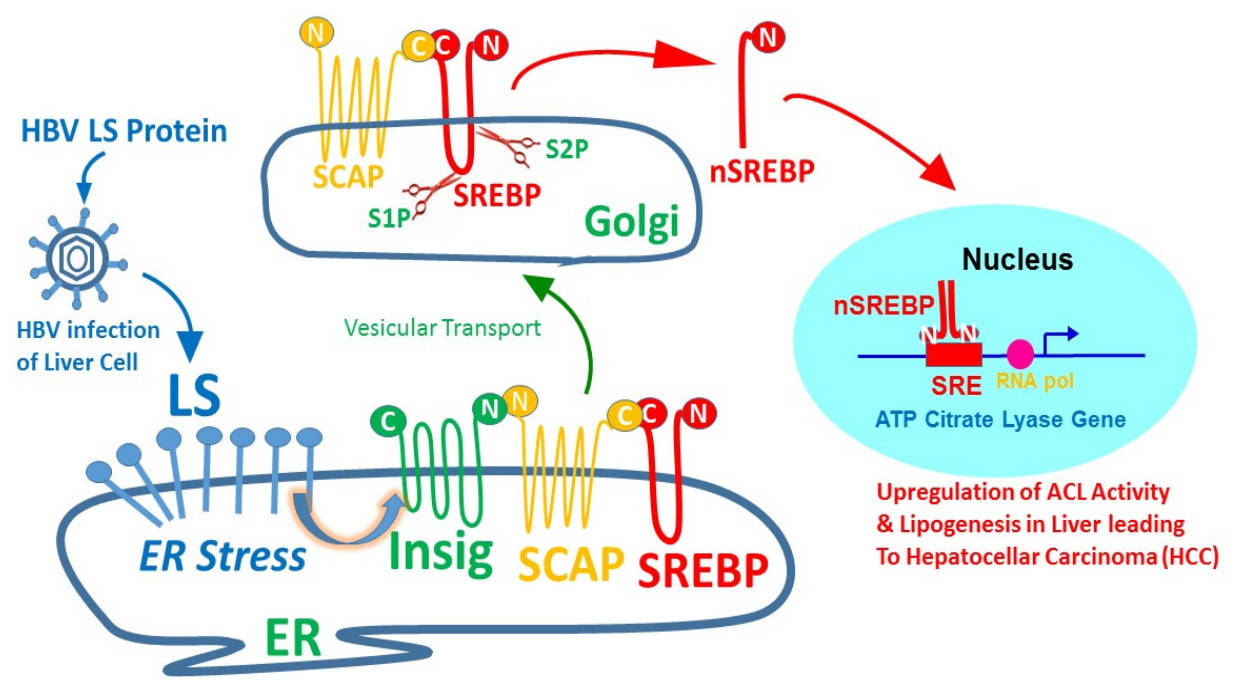

Figure 2. Model for the regulation of Endoplasmic Reticulum (ER) membrane resident Insig/SCAP/SREBP complex by the Hepatitis B Virus Large Surface (LS) protein in hepatocellular carcinoma (HCC) liver cells.

Accumulation of LS protein in the ER of chronic HBV infected liver cells promotes ER stress. The close proximity within the ER membrane of the LS protein and the Insig/SCAP/SREBP complex leads to the interaction of LS with Insig either directly or mediated by other regulatory factor(s), resulting in the dissociation of Insig with the SCAP/SREBP complex. The SCAP/SREBP complex is transported by vesicles to the Golgi, where resident proteases S1P and S2P sequentially cleave the sterol regulatory element-binding protein (SREBP) to release the NH2-terminal domain mature nuclear form, nSREBP. The NH2-terminal domain contains an acidic transcriptional activator motif and a bHLH-Zip motif that is responsible for DNA binding and dimerization of the mature transcriptionally active nSREBP. nSREBP translocates to the nucleus and is targeted as a dimer to the SRE sites of the promoter of the lipogenic ATP citrate lyase (ACL) gene and activates transcription of ACL.

High levels of ACL enzyme activity is believed to lead to promotion of tumorigenesis of the liver cell 
The localization and accumulation of the HBV viral LS protein in the ER membrane in close proximity to the ER transmembrane SREBP proteins may very well provide a mechanistic strategy for the LS protein to activate the ACL gene and promote liver tumorigenesis. I hereby, propose a plausible mechanism by which the LS protein may activate ACL gene activity (Figure 2). I hypothesize that the accumulation of large amounts of the viral LS protein in the ER membrane of the infected liver cell leads to interaction with the membrane bound Insig/SCAP/SREBP complex. The LS protein in close proximity to the SREBP complex may interact via specific protein-protein domain interactions with the Insig protein promoting dissociation of Insig with the SCAP protein. The SCAP protein undergoes a conformational change resulting in this escort protein of ER moving the SREBP/SCAP protein complex into the Golgi vesicle where the S1P and S2P proteases clip the immature inactive form of SREBP into the processed $\mathrm{N}$ terminal domain mature active form of SREBP (nSREBP). The NH2-terminal domain of SREBP contains the bHLH-LZip motif that is responsible for dimerization and binding to target DNA and an acidic motif believed to be the transcriptional activation domain (Cheng et al., 2018). nSREBP translocates to the nucleus mediated by its nuclear localization signal and binds to sterol regulatory elements (SRE) present in the ATP citrate lyase (ACL) gene promoter, thereby elevating ACL transcription levels. This leads to overproduction of the ACL enzyme protein and consequently conversion of glucose to lipids that is necessary for proliferating cells. LS, therefore, may facilitate the tumorigenic process via its effects on the lipogenic biochemical pathway.

\section{Discussion}

Future studies to prove the validity of the proposed model would involve protein-protein interaction experiments using a variety of available approaches to investigate interaction between LS and Insig, including Yeast Two Hybrid system; Co-Immunoprecipitation \& Pull Down; and, Far Western Blot analysis. The LS protein of HBV is composed of three domains: $\mathrm{N}$ - terminal preS1 domain, a central preS2 domain and a $\mathrm{C}$-terminal $\mathrm{S}$ domain. The preS1 and preS2 domains have been shown to be necessary for the oncogenic potential of the LS protein and, therefore, would be expected to contain specific amino acid residues and motifs capable of protein-protein interaction with the Insig protein in the ER resulting in the disruption of Insig interaction with the SCAP-SREBP complex in the ER membrane. The interaction of LS protein with Inisg protein may be a direct protein-protein interaction or may be mediated by additional factors and their discovery would offer more potential strategies for therapeutic intervention of LS activity. The mutant large surface protein with an in-frame deletion in the preS2 region, S2-LHBs has been shown to induce ER stress and also has oncogenic potential. It is likely that this deletion mutant has an altered three-dimensional conformation that may result in a more efficient interaction of the LS surface protein with Insig accelerating the release of Insig from the SCAP-SREBP complex and subsequent vesicular transport of the SCAP-SREBP complex to the Golgi from where the nSREBP transcriptionally active mature form is released.

There are three isoforms of SREBP, namely SREBP-1a, SREBP-1c, and SREBP-2. SREBP-1 was shown to be elevated in HBV positive HCC patients ( $\mathrm{Li}$ et al., 2014). However, there are limited studies on SREBP transcription factor upregulation in liver cells infected with HBV using transgenic mice and cell culture model systems. The studies performed by Churin et al. (2014) using transgenic mouse expressing the LS protein of HBV did not report the status of ACL and SREBP activity. There is one recent report showing that SREBP-1 was elevated in transgenic mouse livers harboring HBV pre-S2 deletion mutant of the LS protein (Teng et al., 2015). It would be important to determine the activity levels of ACL, and identify the specific isoforms of SREBP in transgenic mouse livers expressing the wild-type LS protein of HBV in the absence of the entire HBV genome. Human hepatic cell culture co-transfection assays using plasmid reporter constructs containing the human ACL promoter sequence fused to a gene reporter sequence and an effector expression plasmid construct containing cDNA sequences for the LS protein of HBV would enable investigating ACL promoter activity and SREBP isoform levels, to complement the in vivo transgenic studies. Chromatin Immunoprecipitation experiments would enable analyzing the promoter occupancy of the ACL promoter by SREBP isoforms to prove that indeed SREBPs stimulate ACL activity levels as a result of the expression of the Hepatitis B Virus LS protein. Lipid reprogramming is a hallmark of cancer and involves a complex network of pathways and genes. It is highly likely that ACL may not be the only target of SREBP activation in HBV infected liver cells exhibiting HCC. It would be important to evaluate other known genes involved in lipid biosynthesis by global gene expression profiling of HCC tissue from transgenic mice liver expressing the LS protein (without the presence of HBV in the genome).

The hypothesis that LS protein and its interaction in the ER membrane with the Insig protein is important for the tumor growth and thus responsible for HBV induced HCC can be validated by developing and delivering small molecules that deactivate this viral protein and/or its interaction with the Insig protein and suppress the tumor 
growth. The mechanisms of tumorigenesis caused by HBV in liver of patients exhibiting chronic infection is complex and unclear. A number of distinct pathways involving well known molecules including transcription factors have been reported. Clearly, combinatorial new therapeutic approaches targeting simultaneously several key molecules and signaling pathways is needed to battle this global disease.

\section{Conflict of interests}

The authors declare that there is no conflict of interests regarding the publication of this paper.

\section{References}

Amemiya-Kudo, M., Shimano, H., Hasty, A. H., Yahagi, N., ..., \& Yamada, N. (2002). Transcriptional activities of nuclear SREBP-1a, $-1 \mathrm{c}$, and -2 to different target promoters of lipogenic and cholesterogenic genes. $J$. Lipid Res., 43(8), 1220-35.

Bauer, T., Sprinzl, M., \& Protzer, U. (2011). Immune control of hepatitis B virus. Digestion Dis., 29, 423-433.

Bouchard, M. J., \& Navas-Martin, S. (2011). Hepatitis B and C virus hepatocarcinogenesis: Lessons learned and future challenges. Cancer Lett., 305(2), 123-43.

Chen, C. J., \& Yang, H. I. (2011). Natural history of chronic Hepatitis B REVEALed. J. of Gastroenterol. Hepatol., 26, 628-638.

Chen, C. J., Yang, H. I., Su, J., Jen, C. L., ..., \& REVEAL-HBV Study Group. (2006). Risk of hepatocellular carcinoma across a biological gradient of serum hepatitis B virus DNA level. J. Am. Medic. Assoc., 295, 65-73.

Cheng, C., Geng, F., Cheng, X., \& Guo, D. (2018). Lipid metabolism reprogramming and its potential targets in cancer. Cancer Commun., 38(27).

Cheng, X., Li, J., \& Guo, D. (2018). SCAP/SREBPs Are Central Players in Lipid Metabolism and Novel Metabolic Targets in Cancer Therapy. Curr. Top. Med. Chem., 18(6), 484-493.

Chisari, F. V., Klopchin, K., Moriyama, Taquinelli, T., ..., \& Palmiter, R. D. (1989). Molecular pathogenesis of Hepatocellular carcinoma in Hepatitis B virus transgenic mice. Cell, 59, 1145-1156.

Cho, H. K., Cheong, K. J., Kim, H. Y., \& Cheong, J. H. (2011). Endoplasmic reticulum stress induced by hepatitis $\mathrm{B}$ virus $\mathrm{X}$ protein enhances cyclo-oxygenase 2 expression via activating transcription factor. Biochemical Journal, 435(2), 431-439.

Churin et al. (2014). Pathological impact of hepatitis B virus surface proteins on the liver is associated with the host genetic background. PLoS One, 9(3), e90608.

Churin, Y., Roderfeld, M., \& Roeb, E. (2015). Hepatitis B virus large surface protein: Function and fame. HepatoBiliary Surg. Nutr., 4(1), 1-10.

DeBerardinis, R. J., \& Chandel, N. S. (2016). Fundamentals of cancer metabolism. Sci. Adv., 2, e1600200.

Eberle, D., Hegarty, B., Bossard, P., Ferré, P., \& Foufelle, F. (2004). SREBP transcription factors: Master regulators of lipid homeostasis. Biochimie, 86, 839-848.

Espenshade, P. J. (2006). SREBPs: Sterolregulated transcription factors. Journal of Cell Science, 119, 973-976.

Gabitova, L., Gorin, A., \& Astsaturoa, I. (2013). Molecular Pathways: Sterols and receptor signaling in cancer. Clin. Cancer Res., 19(23), 6344-635.

Gambhir, S. S. (2002). Molecular Imaging of cancer with positron emission tomography. Nat. Rev. Cancer, 2, 683-693.

Granchi, C. (2018). ATP citrate lyase (ACLY) inhibitors: An anti-cancer strategy at the crossroads of glucose and lipid metabolism. European Journal of Medicinal Chemistry, 157, 1276-1291.

Guo, D., Bell, E. H., \& Chakravarti, A. (2013). Lipid metabolism emerges as a promising target for malignant glioma therapy. CNS Oncol., 2(3), 289-299.

Hadziyannis, S., Gerber, M. A., Vissoulis, C., \& Popper, H. (1973). Cytoplasmic hepatitis B antigen in "ground-glass" hepatocytes of carriers. Arch. Pathol., 96, 327-330.

Hajjou, M., Norel, R., Carver, R., Marion, P., ..., \& Rogler, C. E. (2005). cDNA microarray analysis of HBV transgenic mouse liver identifies genes in lipid biosynthetic and growth control pathways affected by HBV. $J$ Med. Virol., 77(1), 57-65. 
Hatzivassiliou, G., Zhao, F., Bauer, D. E., Andreadis, C., ..., \& Thompson, C. B. (2005). ATP citrate lyase inhibition can suppress tumor cell growth. Cancer Cell, 8(4), 311-21.

Hollinger, F. B. (1996). Hepatitis B virus. In B. N. Fields, D. M. Knipe, \& P. M. Howley (Eds.), Fields' virology (pp. 2739-2807). Lippincott-Raven Publishers, Philadelphia, Pa.

Horton, J. D., Goldstein, J. L., \& Brown, M. S. (2002). SREBPs: Activators of the complete program of cholesterol and fatty acid synthesis in the liver. J. Clin. Invest., 109, 1125-1131.

Hsieh et al. (2004). Pre-S mutant surface antigens in chronic hepatitis B virus infection induce oxidative stress and DNA damage. Carcinogenesis, 25, 2023-2032.

Jacquemyn, J., Cascalho, A., \& Goodchild, R. E. (2017). The ins and outs of endoplasmic reticulum-controlled lipid biosynthesis. EMBO Reports, 18(11), 1905-1921.

Kadowaki, T., Makuuchi, M., Ohnishi, S., Osuga, J., \& Yamada, N. (2005). Co-ordinate activation of lipogenic enzymes in hepatocellular carcinoma. Eur. J. Cancer., 41(9), 1316-22.

Lamontagne, R. J., Bagga, S., \& Bouchard, M. J. (2016). Hepatitis B virus molecular biology and pathogenesis. Hepatoma Res., 2, 163-186.

Lazar, C., Uta, M., \& Branza-Nichita, N. (2014). Modulation of the unfolded response by the human hepatitis B virus. Frontiers in Microbiology, 5, 433.

Lee, H. J., Kim, H., Lee, S. A., Won, Y. S., ..., \& Kim, B. J. (2015). Upregulation of endoplasmic reticulum stress and reactive oxygen species by naturally occurring mutations in hepatitis B virus core antigen. Journal of General Virology, 96, 1850-1854.

Levrero, M., \& Zucman-Rossi, J. (2016). Mechanisms of HBV-induced hepatocellular carcinoma. J. Hepatol., 64.

Li, C., Yang, W., Zhang, J., Zheng, X., Yao, Y., Tu, K., \& Liu, Q. (2014). SREBP-1 Has a Prognostic Role and Contributes to Invasion and Metastasis in Human Hepatocellular Carcinoma. Int. J. Mol. Sci., 15, 7124-7138.

Li, Y. W., Yang, F. C., Lu, H. Q., \& Zhang, J. S. (2016). Hepatocellular carcinoma and hepatitis B surface protein. World J Gastroenterol., 22(6), 1943-1952.

Mani, S. K., \& Andrisani, O. (2018). Hepatitis B Virus-Associated Hepatocellular Carcinoma and Hepatic Cancer Stem Cells. Genes, 9, 137.

Misawa, K., Horiba, T., Arimura, N., Hirano, Y., ..., \& Sato, R. (2003). Sterol regulatory element-binding protein-2 interacts with hepatocyte nuclear factor-4 to enhance sterol isomerase gene expression in hepatocytes. J. Biol. Chem., 278(38), 36176-82.

Montalbano, R., Honrath, B., Wissniowski, T. T., Elxnat, M., ..., \& Fazio, P. D. (2016). Exogenous hepatitis B virus envelope proteins induce endoplasmic reticulum stress: Involvement of cannabinoid axis in liver cancer cells. Oncotarget, 7(15).

Moon, Y. A., Lee, J. J., Park, S. W., Ahn, Y. H., \& Kim, K. S. (2000). The roles of sterol regulatory element-binding proteins in the transactivation of the rat ATP citrate-lyase promoter. J. Biol. Chem., 275(39), 30280-6.

Moslehi, A., \& Hamidi-zad, Z. (2018). Role of SREBPs in Liver Diseases: A Mini-review. Journal of Clinical and Translational Hepatology, 6, 332-338.

Neuveut, C., Wei, Y., \& Buendia, M. A. (2010). Mechanisms of HBV-related hepatocarcinogenesis. J. of Hepatology, 52, 594-604.

Park, S., Moon, Y., Kim, K., Ahn, Y., \& Kim, Y. (1997). Cloning and characterization of the 5' flanking region of human ATP-citrate lyase gene. Biochi.m Biophys. Acta., 1353(3), 236-40.

Pollicino, T., Cacciola, I., Saffioti, F., \& Raimondo, G. (2014). Hepatitis B virus PreS/S gene variants: Pathobiology and clinical implications. Journal of Hepatology, 61, 408-417.

Ren, F., Shi, H., Zhang, L., Zhang, X., ..., \& Duan, Z. (2016). The dysregulation of endoplasmic reticulum stress response in acute-on-chronic liver failure patients caused by acute exacerbation of chronic hepatitis B. $J$. Viral Hepat., 23(1), 23-31.

Riha, R., Gupta-Saraf, P., Bhanja, P., Badkul, S., \& Saha, S. (2017). Stressed Out-Therapeutic Implications of ER Stress Related Cancer Research. Oncomedicine, 2. 
Ringehan, M., McKeating, J. A., \& Protzer, U. (2017). Viral hepatitis and liver cancer. Phil. Trans. R. Soc. B, 372, 20160274.

Riviere, L., Ducroux, A., \& Buendia, M. A. (2014). The Oncogenic role of Hepatitis B virus. In M. H. Chang \& K. T. Jeang (Eds.), Viruses and Human Cancer, Recent results in cancer research 193. Springer Verlag, Berlin Heidelberg.

Sato, R., Okamoto, A., Inoue, J., Miyamoto, W., ..., \& Maeda, M. (2000). Transcriptional Regulation of the ATP Citrate-lyase Gene by Sterol Regulatory Element-binding Proteins. J. Biol. Chem., 275(17), 12497-12502.

Seeger, C., \& Mason, W. S. (2015). Molecular Biology of Hepatitis B virus infection. Virology, 479-480, 672-686.

Shao, W., \& Epenshade, P. J. (2012). Expanding roles for SREBP in metabolism. Cell. Metab., 16, 414-419.

Shimano, H., Yahagi, N., Amemiya-Kudo, M., Hasty, A. H., ..., \& Yamada, N. (1999). Sterol Regulatory Element-binding Protein-1 as a Key Transcription Factor for Nutritional Induction of Lipogenic Enzyme Genes. J. Biol. Chem., 274, 35832-35839.

Shimomura, I., Shimano, H., Korn, B. S., Bashmakov, Y., \& Horton, J. D. (1998). Nuclear sterol regulatory element-binding proteins activate genes responsible for the entire program of unsaturated fatty acid biosynthesis in transgenic mouse liver. J. Biol. Chem., 273, 35299-35306.

Song, J., Zhang, X., Ge, Q., Yuan, C., ..., \& Zhang, B. (2018). CRISPR/Cas9-mediated knockout of HBsAg inhibits proliferation and tumorigenicity of HBV-positive hepatocellular carcinoma cells. J Cell Biochem., $1-13$.

Teng, C. F., Wu, H. C., Hsieh, W. C., Tsai, H. W., \& Su, I. J. (2015). Activation of ATP Citrate Lyase by mTOR Signal Induces Disturbed Lipid Metabolism in Hepatitis B Virus Pre-S2 Mutant Tumorigenesis. J. of Virology, 89(1).

Teng, C. F., Wu, H. C., Shyu, W. C., Jeng, L. B., \& Su, I. J. (2017). Pre-S2 Mutant-Induced Mammalian Target of Rapamycin Signal Pathways as Potential Therapeutic Targets for Hepatitis B Virus-Associated Hepatocellular Carcinoma. Cell Transplantation, 26, 429-438.

Wang, H. C., Huang, W., Lai, M. D., \& Su, I. J. (2006). Hepatitis B virus pre-S mutants, endoplasmic reticulum stress and hepatocarcinogenesis. Cancer Science, 97(8), 683-688.

Wang, H., Kouri, G., \& Wollheim, C. B. (2005). ER stress and SREBP-1 activation are implicated in beta-cell glucolipotoxicity. Journal of Cell Science, 118, 3905-3915.

Xu, H. Z., Liu, Y. P., Guleng, B., \& Ren, J. L. (2014). Hepatitis B Virus-Related Hepatocellular Carcinoma: Pathogenic Mechanisms and Novel Therapeutic Interventions. Gastrointest. Tumors, 1, 135-145.

Xu, Z., Jensen, G., \& Benedict Yen, T. S. (1997). Activation of Hepatitis B Virus S Promoter by the Viral Large Surface Protein via Induction of Stress in the Endoplasmic Reticulum. J. of Virology, 71(10), 7387-7392.

Yahagi, N., Shimano, H., Hasegawa, K., Ohashi, K., ..., \& Yamada, N. (2005). Co-ordinate activation of lipogenic enzymes in hepatocellular carcinoma. Eur. J. Cancer., 41(9), 1316-22.

Zaidi, N., Royaux, I., Swinnen, J. V., \& Smans, K. (2012). ATP Citrate Lyase Knockdown Induces Growth Arrest and Apoptosis through Different Cell- and Environment Dependent Mechanisms. Mol. Cancer Ther., 11(9).

\section{Copyrights}

Copyright for this article is retained by the author(s), with first publication rights granted to the journal.

This is an open-access article distributed under the terms and conditions of the Creative Commons Attribution license (http://creativecommons.org/licenses/by/4.0/). 